\title{
Materials science challenges in paintings
}

Philippe Walter and Laurence de Viguerie

Through the paintings of the old masters, we showcase how materials science today provides us with a vision of the processes involved in the creation of a work of art: the choice of materials, the painter's skill in handling these materials, and the perception of the finished work.

Recent advances in methods of chemical analysis, including radiation physics and spectroscopy, have led to new considerations on the role that materiality plays in the process of the artistic creation of a painting. While until recently it was essential to take samples in order to analyse a painting, it has become possible to construct miniaturised instruments that can be moved into museums and historical sites to non-invasively identify the components of the works of art. X-rays, with methods such as X-ray fluorescence spectroscopy and X-ray diffraction, and lasers, with Raman spectrometry and laser-induced fluorescence spectrometry, for example, today play an important role in these new areas of scientific investigation. ${ }^{1}$ Thus, we can observe the rising of a new field combining analytical chemistry, materials science and art history, which is sometimes called "technical art history". Thanks to the scientific characterisation of painting materials under different radiations (X-rays, UV, visible and infrared light, and $\mathrm{THz}$ ), and physicochemical considerations, it is possible to identify the components in the paint layers, to understand the gestures of artists, to make visible earlier stages of the realisation of the painting, and to reveal alteration of the images, i.e. pentimenti, when the painter has changed the composition during the process of painting. All these aspects contribute to a better understanding of how artists had to deal with their materials in order to achieve the project they had in mind. Furthermore these data are useful for curators and art conservators when they have to make the right choice during painting treatments or when it is necessary to consider colour alterations of the pigments that modified the appearance of the paintings.

The history of arts, particularly the history of painting, has often shown the crucial role played by the proper handling of pigments and binders. Whereas Leonardo da Vinci was able to use very thin layers of a dark glaze to create the face of the Mona Lisa as has been proved by chemical analyses ${ }^{2}$, he failed in several other projects, including the famous representation of The Battle of Anghiari, which was to decorate the Hall of the Five Hundred in the Palazzo Vecchio in Florence. ${ }^{3,4}$ The reasons for this failure have not yet been completely understood: his biographers reported inappropriate formulation and paint manipulation that led to the painting flowing down the wall instead of fixing itself on its surface. Leonardo's manuscripts and paintings nevertheless show that he was an exceptional experimenter, convinced of the importance of grinding minerals to produce pigments and extracting oils, gums, resins and spirits from plants and trees to formulate complex binders and varnishes. Later, other painters felt themselves to be disadvantaged by the limitations induced by the use of the pigments and binders they had at their disposal, asking themselves questions about their artistic practices because they were persuaded of the necessity of acquiring more chemical or alchemical 
knowledge to make the best choices during the realisation of the paintings. For example, Vincent Van Gogh (fig. 1) wrote to his brother Theo, when he was in Auvers-sur-Oise on June 17, 1890: "In the colours there is adulteration as in wines. How can one judge correctly when, like myself, one knows nothing of chemistry." 5

The importance of materials in artistic creation can also be seen in the $20^{\text {th }}$ century, and still today, when artists advertise widely their acquisition of the exclusive use of a colour. For example, at the end of the 1950s, Yves Klein asked his paint supplier Edouard Adam, installed in Paris, to formulate a new colour, with a "secret" composition that he could protect, as one does inventions. After many trials, Adam obtained a mixture composed of synthetic ultramarine blue pigment and the polyvinyl acetate binder Rhodopas-M put on the market in 1956 by the Rhone-Poulenc Company. ${ }^{6}$ This "International Klein Blue" - or IKB - will then become a differentiating element for the artist and will allow him to invent new ways of painting, especially with sponges and using nude females as "living paintbrushes". More recently, in 2016, Anish Kapoor acquired the exclusive rights for a new synthetic pigment, the Vantablack S-Vis, invented by the English company Surrey NanoSystems and made up of carbon nanotubes assembled in a structure similar to that of coral, which has the property of being a deep black reflecting less than $0.3 \%$ of visible light. ${ }^{7}$ Anish Kapoor stressed again to the public the fundamental role played by Materials Science during the process of artistic creation.

But this behaviour is not new. The methods used for synthesising new pigments that do not exist in Nature is a very ancient part of the intangible heritage of mankind: for example, the lack or scarcity of pleasing natural blue pigments induced the invention of artificial inorganic blue minerals in Egypt or Mesopotamia during the $3^{\text {rd }}$ millennium B.C. and in China ${ }^{8}$ during the $1^{\text {st }}$ millennium B.C. as well as hybrid organic-inorganic blue by the Maya civilization ${ }^{9}$. Wet chemistry, thermal recrystallisation and related annealing processes were at the origin of such syntheses and were applied to develop a large number of colours that were adopted by artists only if they were intense, pure, vivid, easily handled with a brush, and stable in light and to the atmosphere. These ancient practices are nowadays source of ideas for designing new pigments with a wide range of properties that can be of interest for industrial and forensics applications. ${ }^{10}$

Over the course of the past two decades, scholars have considered the necessity and the benefits of analytical chemistry 11,12 that allows new in-situ measurements with non invasive spectroscopies, chemical mapping to reveal hidden paintings as well as high spatial resolution identification of materials with microfocussed beams of synchrotron X-rays to identify complex mixtures and alteration phenomena. Using multivariate analysis and chemometrics to interpret the large sets of data obtained by imaging spectroscopy, 13,14 they have demonstrated that the identification of one specific pigment or substance in a complex recipe contributes to the understanding of the evolution of artistic practices and is helpful for the conservation of works of art and for their authentication. More recently new approaches are dealing with materials science and are aiming at a better understanding of the theoretical aspects of painting materials and of their behaviour as mixtures of pigments and binders. In this Commentary we will discuss examples that serve to illustrate the need for a better understanding of the properties of artist materials.

\section{Innovations in the materials of painters}

More than the selection of pigments of natural or artificial nature, the evolution in their formulation with binders illustrates how it was important for painters to consider the 
interactions between the components that are at the origin of the artistic effects they wished to achieve. Creating light and shadow, and the effects of movement are key factors in the successful realisation of a painting that has long been trying to create a mimetic and sensitive representation of Nature. Our brain's perception of light, relief and motion in the painting is largely controlled by the addition of white or dark pigments, and by the use of prominent brushstrokes that can generate an image that is an outstandingly realistic representation of how light moves. On the materials side, that means that the capability to add substances that confer specific physical properties to a paint material constituted a crucial challenge that simultaneously provided new aesthetic opportunities to the artists. But it is difficult to determine what was the driving force behind innovation: materials science or art? The following two examples will illustrate the search for translucency and plasticity.

Since the end of the $15^{\text {th }}$ century, painters have preferred oil painting because siccative oils dried more slowly and uniformly than the organic binders used before (such as egg, glue, casein and gum Arabic) and because it can be easily superimposed to mask previous colours. These two qualities allowed artists plenty of time for correcting and further elaborating their works. Materials for oil painting were then prepared in artists' workshops by grinding pigments with linseed, walnut or poppy oil (often pre-treated with lead oxide to improve the siccative properties) to produce a paste-like material that could be easily applied with a brush. When more binder was added to create what is called a glaze, the material became more fluid, its drying-time longer, and the final result translucent and displaying exceptional colour saturation. ${ }^{15}$ Tuning the translucency of layers was then used to produce subtle effects. Fifteenth- and sixteenth-century Italian and Dutch painters mixed colourless powdered glass in paint layers for its optical properties and as a siccative for oil. ${ }^{16,17}$ Leonardo da Vinci used the glazing technique to create shadows on the face of Mona Lisa ${ }^{2}$, whereas Rembrandt added starch grains ${ }^{18}$ and Nicolas Poussin a fine calcite powder ${ }^{19}$ to the paint applied - in the case of the latter two artists - on coloured grounds. Calcite and starch have refractive indices close to that of dried siccative oils, thus increasing the translucency of the paint layers, allowing the transfer of light down to the deepest layers.

Another important challenge for artists was to tailor the viscosity behaviour of the paint material to obtain smooth or rough surfaces, and to avoid any mixing of the colours during their work. Rheological studies on reconstitutions of old formulas have shown how the amount of oil or the adding of a resin-based medium mixed with the oil can be helpful to tune the brushability and the capability of the paint to level itself out or, on the contrary, to retain the marks of the brush. ${ }^{20}$ Paints close to the Newtonian behaviour of oil were used as glazes since the $15^{\text {th }}$ century before artists started to use pseudoplastic or even thixotropic mixtures. At the beginning of the 19th century, chemists introduced to art gel-type mediums such as gumtion, consisting of a mixture of oil, lead acetate and mastic resin. These hybrid gels exhibit a lamellar type structure in a continuous phase. ${ }^{21}$ When mixed with oil paint, even at low concentrations, the elastic and loss moduli of the paint material are enhanced as well as the yield stress value: this induces the formation of a gel with stronger elastic properties than the gumtion itself. Such an effect confirms the attractiveness of this medium to painters, allowing them to paint fast and to create high impastos.

The pigment type, its graininess and concentration also influence the rheological properties of the paint. It was observed that lead white and zinc white oil paint exhibit similar rheological behaviours, with a solid to fluid transition, but zinc white allows 
higher impasto when formulated with the same oil content (fig. 2). ${ }^{22}$ Nevertheless, as is known in the modern industrial paint industry, all these systems exhibit a complex behaviour with possible time-dependent effects, and it is difficult to predict with precision their evolution after application. Van Gogh took advantage of these complex properties during the creation of his works, using one or other of the white pigments in specific areas of the painting, and he commented - using his own vocabulary - the importance of the rheological properties of the paint film in a letter to his brother: "What a funny thing the touch is, the brushstroke. Out of doors, exposed to the wind, the sun, people's curiosity, one works as one can, one fills one's canvas regardless. Yet then one catches the true and the essential - that's the most difficult thing. But when one returns to this study again after a time, and orders one's brushstrokes in the direction of the objects - certainly it's more harmonious and agreeable to see, and one adds to it whatever one has of serenity and smiles. $»^{23}$

\section{Highlighting the origin of visual perception}

Reflection, transmission, and absorption of light by paint layers are also of great importance for artists. Explanation of the origin of colour in a painting is a new and attractive direction of research because chemical analyses of works of art are providing the exact nature of more or less all the components in the paint layers. In the case of condensed matter such as paint films, multiple scattering events are taking place: coloration depends on film thickness, pigment concentration, and granularity.

When considering dyeing molecules that have been used since Antiquity for synthesizing lake pigments by their precipitation or adsorption onto insoluble and white salts, calculations of their interaction with light can be obtained from first principles and density-functional theory (DFT) approach, as demonstrated for alizarin and its alkali metal complex compounds in a solvent. ${ }^{24}$ Colour analysis validates the constituents and the geometrical structures of the model complexes used for the calculations.

For a pigment alone, theoretical considerations can also be used to calculate the interaction of paint with light. Electronic-structure calculations by DFT were carried out to investigate the origin of the complex structure of the colour-transition edges in the semiconductor pigments vermillion $(\alpha-\mathrm{HgS})$, minium $\left(\mathrm{Pb}_{3} \mathrm{O}_{4}\right)$ and lead-tin yellow $\left(\mathrm{SnPb}_{2} \mathrm{O}_{4}\right)^{25} \mathrm{~A}$ fairly small frequency range above the absorption edge determines their colour. But their nano- and micro- structures also play an important role in their appearance because the propagation of light inside a granular material depends on the absorption of light by the particles or the matrix, and on the scattering of light by the particles. Tomczak et al. ${ }^{26}$ have suggested the use of a methodology combining electronic structure and many-body techniques to describe compact powders of pigments. These models are convenient for describing the specific colours of pigments and to provide a quantitative criterion for the evaluation of pigment performances. The bright red colour vermilion is due to the fact that its reflectance curve drops quickly, so that the yellow to blue parts of the spectrum are suppressed (fig. 3).

In the case of a real painting, the complexity of the absorption and scattering phenomena that occur during interaction between light and paint, arises also from the nature of the binder, the mixing of ingredients, the superimpositions of layers applied to the canvas, the irregularities of the painting surface, etc. It is then very challenging to deduce a physical model describing such a complex system from descriptions of the ingredients. Assuming that the material is isotropic, non-fluorescent, non-glossy and the 
sample is illuminated by diffuse light, a simplified analysis of the interaction of incoming light with a layer of opaque paint can be obtained with the Kubelka-Munk model (KM). The absorption and scattering coefficients of paint are expressed in relation to its overall reflectance. Various implementations and extensions of the KM theory are used for pigment mixture identification using spectral reflectance measurements and for the characterisation of stacks of scattering and non-scattering films. ${ }^{27}$ These approaches can then be implemented to understand why an artist has mixed pigments in various proportions to produce different hues. ${ }^{28}$

This progress on the theoretical calculation of colour in complex paintings has been made only very recently. The interest of this research also resides in the possibility to exploit the influence of interaction with light to highlight the evolution of paintings through time, when this interaction has disturbed the appearance of the image. Many chemical reactions have occurred in pigments within the paint film, for example, in paintings by Van Gogh, on lead chromates, ${ }^{29}$ cadmium sulphides, ${ }^{30}$ red lead ${ }^{31}$ or eosinbased geranium lakes. ${ }^{32,33}$ Theoretical considerations and experiments using an electrochemical set-up mimicking the real environment ${ }^{34}$ have been suggested to help understand the basic features of chemical kinetics for solid-solid transformations that it is important to consider when we try to reconstitute the original colours of paintings. ${ }^{32,35}$ Digital reconstructions of the original aspects of the painting are becoming a new and appealing application of the studies of painting materials and artists' practices.

Philippe Walter* and Laurence de Viguerie are at the Laboratoire d'archéologie moléculaire et structurale (LAMS), Sorbonne Universités, UPMC Univ Paris 06, CNRS, UMR 8220, 4 place Jussieu 75005 Paris, France.

*e-mail:philippe.walter@upmc.fr; laurence.de viguerie@upmc.fr.

\section{References}

${ }^{1}$ Brunetti B. G., Miliani C., Rosi F., Doherty B., Monico L., Romani A. \& Sgamellotti A, Top Curr Chem (Z) 374:10, DOI 10.1007/s41061-015-0008-9 (2016).

2 de Viguerie L, Walter P., Laval E., Mottin B. \& Solé V.A., Angew. Chem. Int. Ed. 49, 61256128 (2010).

3 Walter P., European Review, 21(2), 175-189 (2013).

4 Seracini M., Faggioni P.R. \& Pancari M.G., Redicea, Rivista interdisciplinare di studi medicei 11, 4-9 (2011).

5 Vincent Van Gogh, Letter 889 to Theo van Gogh. Auvers-sur-Oise, Tuesday, 17 June 1890, http://vangoghletters.org/vg/letters/let889/letter.html

${ }^{6}$ Adam E., Itinéraire d'un marchand de couleurs, Editions du Chêne, 240 p. (2011).

${ }^{7}$ SurreyNanosystem website; https://www.surreynanosystems.com/assets/media/ vantablack-s-vis-a4-data-brochure-2016-025-download.pdf

8 Berke H., Chem. Soc. Rev. 36, 15-30 (2007).

9 Doménech A., Doménech-Carbó M.T., Vidal-Lorenzo C. \& Vàzquez de Agredos-Pascual M.L., Angew. Chem. Int. Ed. 51, 700 -703 (2012).

10 Erington B., Lawson G., Lewis S.W. \& Smith G. D., Dyes and Pigments 132, 310-315 (2015). 
11 Janssens K., Dik J., Cotte M. \& Susini J., Accounts of Chemical Research 43(6), 814-825 (2010).

12 Mazzeo R. (Ed.), Analytical Chemistry for Cultural Heritage, Topics in Current Chemistry Collections, 364 p. (2017).

13 Cucci C., Delaney J. K., \& Picollo M., Acc. Chem. Res., 2016, 49 (10), pp 2070-2079

14 Pouyet E., Fayard B., Salomé M., Taniguchi Y., Sette F. \& Cotte M., Heritage Science, 3:3 (2015)

15 Elias M \& Simonot L., Applied Optics 45(13), 3168-3172 (2006).

16 Spring M. Heritage Science 5:40 (2017).

17 Roy A, Spring M \& Plazzotta C., Natl. Gall. Tech. Bull., 25, 4-35 (2004).

18 Sanyova J., Cersoy S., Richardin P., Laprevote O., Walter P. \& Brunelle A., Anal. Chem. 83, 753-760 (2011).

19 Noun M., Van Elslande E., Touboul D., Glanville H., Bucklow S., Walter P. \& Brunelle A., J. Mass Spectrometry 51, 1196-1210 (2016).

20 de Viguerie L., Ducouret G., Lequeux F., Moutard-Martin T. \& Walter P., C. R. Physique, 10, 612-621 (2009)

21 de Viguerie L. de, Jaber M., Pasco H., Lalevée J., Morlet-Savary F., Ducouret G., Rigaud B., Pouget T., Sanchez C. \& Walter P., Angew. Chem. Int. Ed., 56(6), 1619-1623, (2017).

22 Salvant-Plisson J., Viguerie L. de, Tahroucht L., Menu M. \& Ducouret G., Colloids and Surfaces A: Physicochemical and Engineering Aspects 458(20), 134-141 (2014).

23 Vincent Van Gogh, Letter 801 to Theo Van Gogh, Saint-Rémy-de-Provence, Tuesday, 10 September 1889, http://vangoghletters.org/vg/letters/let801/letter.html

24 Jeliński T. \& Cysewski P., J Mol Model. 22(6),126-135 (2016).

25 Pallipurath A.R., Skelton J.M., Ricciardi P. \& Elliott S.R., Talanta 154, 63-72 (2016).

26 Tomczak J.M., Pourovskii L.V.,Vaugier L., Georges A. \& Biermann S., Proc. Nat. Acad. Sci. 110, 904-907 (2013).

27 Hebert M. \& Emmel P., in M. Kriss, Handbook of Digital Imaging, 2, Wiley, 1234-1278, (2015)

28 Berns R.S. \& Mohammadi M., Color Res. Appl. 32, 201-207(2007)

29 Monico L., Janssens K., Hendriks E., Vanmeert F., Van der Snickt G., Cotte M. Falkenberg G., Bruneti B.G., Miliani C., Angew. Chem. Int. Ed. 127(47), 14129-14133 (2015).

30 Van der Snickt G., Janssens K., Dik J., De Nolf W., Vanmeert F., Jaroszewicz J., Cotte M., Falkenberg G. \& Van der Loeff L., Anal. Chem. 84, 10221-10228 (2012).

31 Vanmeert F., Van der Snickt G. \& Janssens K., Angew. Chem. Int. Ed. 54, 3607 -3610 (2015).

${ }^{32}$ Fieberg J.E., Knutas P., Hostettler K. \& Smith G.D., Applied Spectroscopy:seppi71(5), 794808 (2017).

33 Centeno S.A., Hale C., Carò F., Cesaratto A., Shibayama N., Delaney J., Dooley K., van der Snickt G., Janssens K. \& Stein S.A., Heritage Science 5 :18 (2017)

34 Anaf W. Trashin S., Schalm O., van Dorp D., Janssens K. \& De Wael K., Anal. Chem. 86(19), 9742-9748 (2014).

35 Cuellar S., Stenger J., Gschwind R., Mohan A., Mukaigawa Y., Raskar R., Eremin K. \& Khandekar N., in ICOM 2011 conference, Lisbon, CD-Rom (2011); and http://www.harvardartmuseums.org/visit/exhibitions/4768/mark-rothkosharvard-murals 


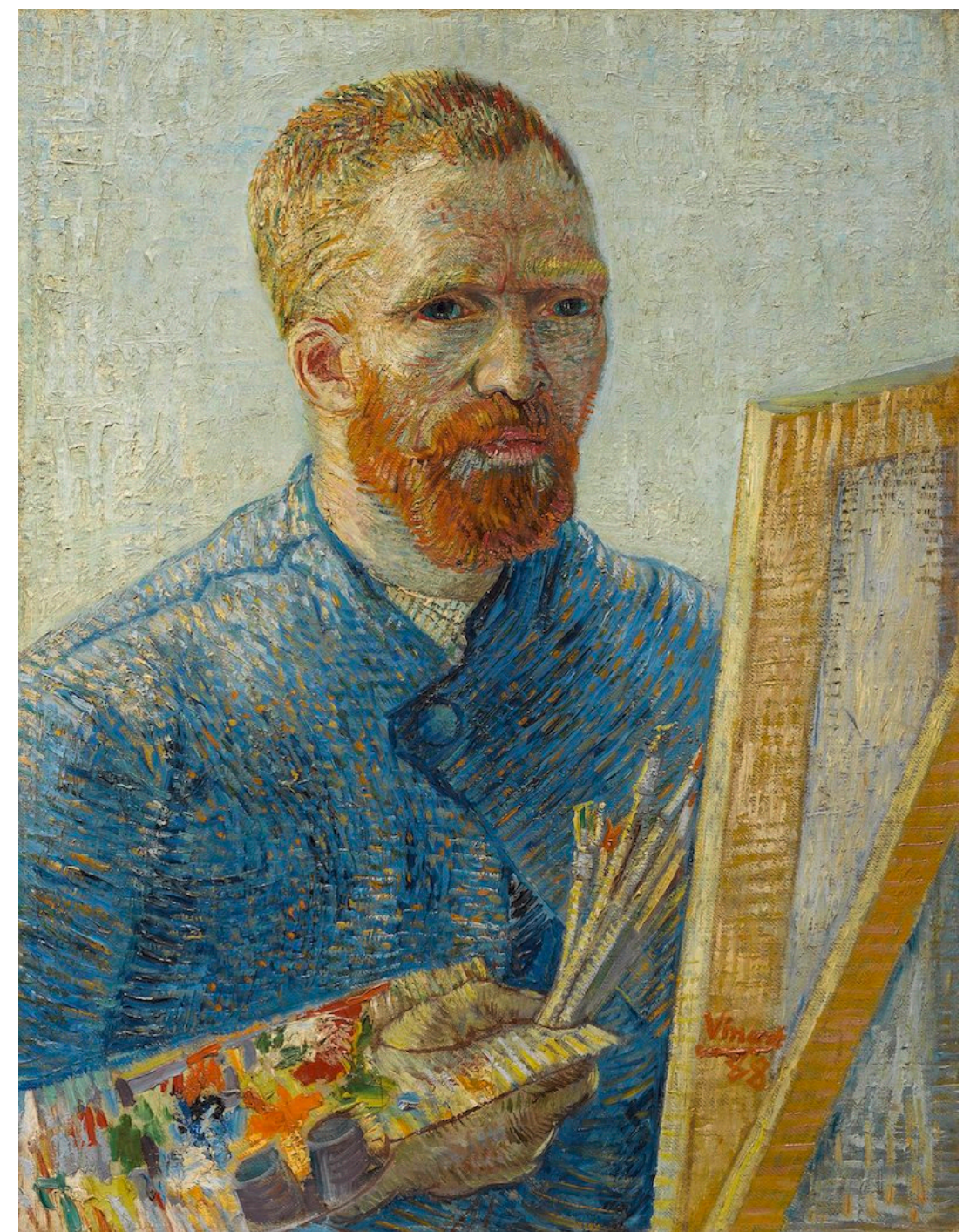

Figure 1 : Vincent Van Gogh, Self-portrait as an artist (1888, Oil on canvas, Van Gogh Museum, Amsterdam). The two containers on the palette most likely contained oil, medium or solvent, with which to adjust the rheological properties of the paint while working on the canvas. 


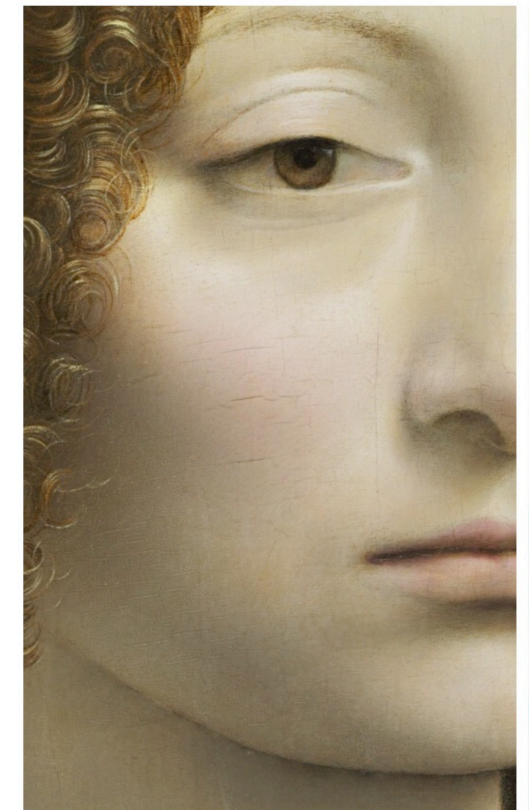

(a)

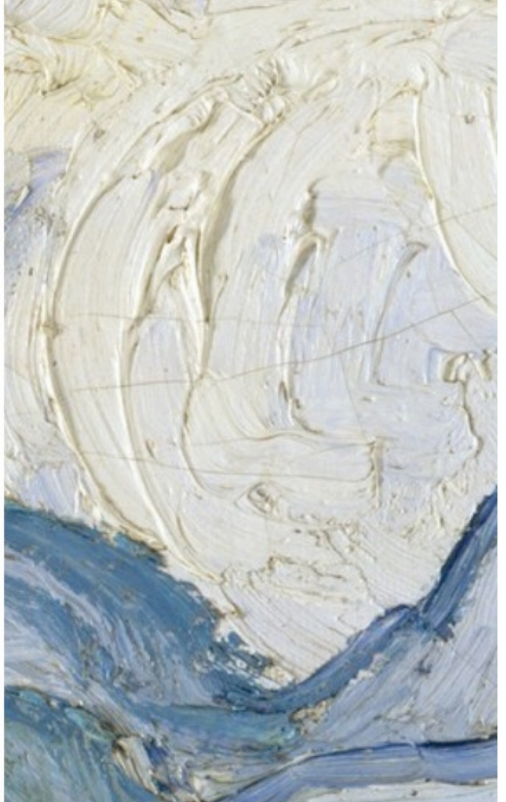

(b)

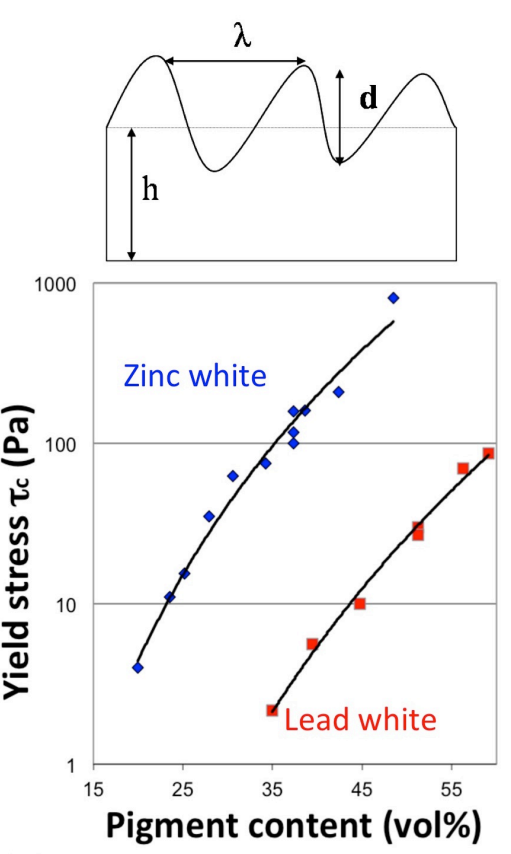

(c)

Figure 2: Details of the same size (circa $12 \times 6 \mathrm{~cm}^{2}$ ) of two paintings: (a) Leonardo da Vinci, Ginevra de' Benci (1474-1478, National Gallery of Art, Washington DC) and (b) Vincent Van Gogh's Wheat Field with Cypresses (1889, Metropolitan Museum of art, New York). Leonardo's painting only displays age cracks and no visible brushstrokes, whereas impasto of considerable thickness is visible in Van Gogh's painting. (c) Model of the paint layer irregularities, with $d$ their depth, $\lambda$ the brush marks width and $h$ the layer thickness, and measurements of the dynamic yield stress $\tau_{c}$ as a function of volume fraction of pigments, for zinc white and lead white oil paints. A low yield stress induces a smooth surface. The yield stress $\tau_{\mathbf{c}}$ and the elastic modulus at $1 \mathrm{~Hz}$ vary in function of the volume fraction of the pigment with power laws (shown with lines) (from ref 22). 


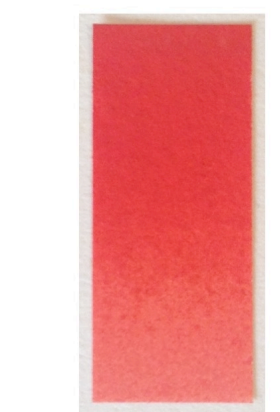

(a) Vermilion
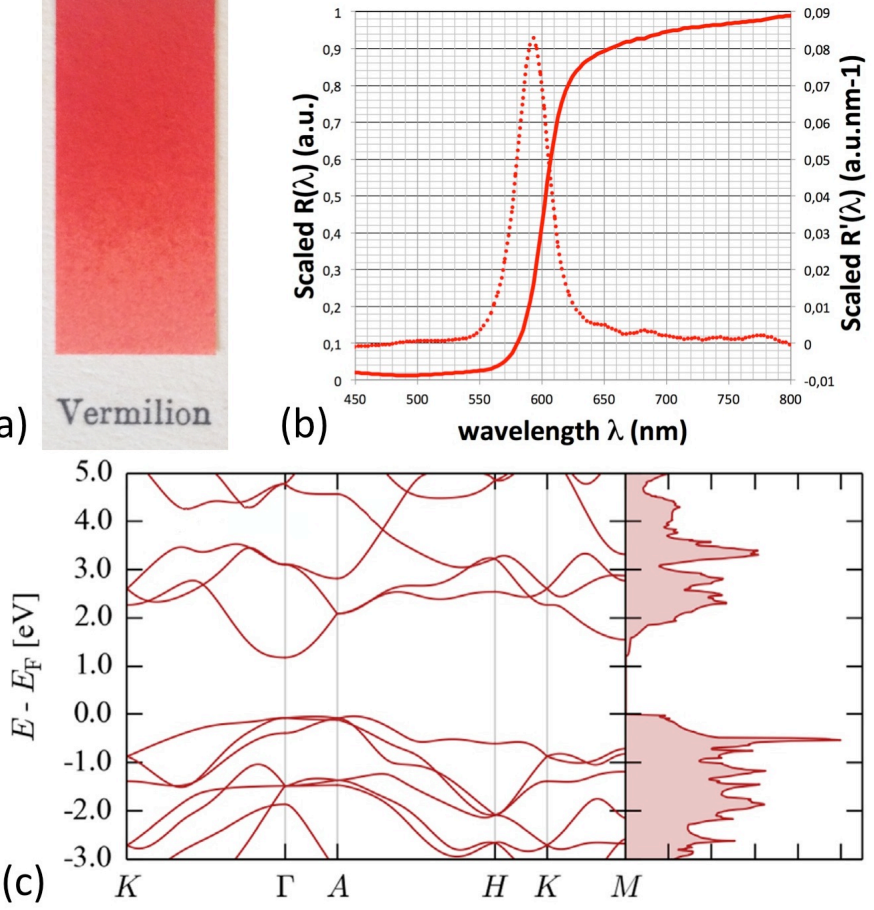

Figure 3: (a) Vermilion watercolour painted on paper, from the Winsor \& Newton Specimen Washes of Artists Water Colours published in 1928. Vermilion is the red pigment containing $\alpha$-HgS (trigonal type), with a very high mean refractive index of 3.2. (b) Its reflectance (continuous line) and derivative (dotted line) spectra measured by fiber optics reflectance spectroscopy (FORS). (c) Electronic band dispersions and density of states curves from density-functional theory (DFT) calculations, showing multiple transition edges in the reflectance spectra (from ref 25) 